\title{
Schistosomal genital granulomas: a report of 10
} cases

\author{
V RATNAM ATTILI, SUBHASH K HIRA, AND M K DUBE \\ From the University Teaching Hospital, Lusaka, Zambia
}

SUMMARY Genital lesions caused by Schistosoma spp are difficult to diagnose because of their similarity to those of common sexually transmitted diseases (STD). Over two years 10 cases of genital lesions were identified by skin or cervical biopsies as being caused by schistosomal infection.

Definitive diagnosis is possible only when schistosomal ova are identified by histological examination of biopsy specimens. Routine biopsies of proliferative genital lesions are therefore recommended in regions where Schistosoma spp are endemic.

\section{Introduction}

Infection of the urinary tract with Schistosoma haematobium and of the lower intestine with Schistosoma mansoni are found in varying proportions in different parts of Zambia. ${ }^{1-4}$ Granulomas developing around ova deposited in the subcutaneous or mucous layers of genital organs by adult worms which have strayed from their primary habitat are not uncommon, especially in women. ${ }^{5-10}$ Such cases are of special interest to venereologists as well as gynaecologists since lesions may easily be mistaken for more common sexually transmitted diseases (STD). Over a period of two years we identified 10 cases (table) of schistosomal genital granulomas in women attending the STD clinic in Lusaka, Zambia, of which these two case reports are typical of their presentation.

\section{Case reports}

CASE 1

A married girl aged 15 years presented with painful enlargement of the clitoris of more than one year's duration. On examination she had a grossly hypertrophic clitoris with an eroded granular surface (fig 1). It was firm and tender to the touch. The inguinal glands were enlarged bilaterally and were firm but not tender. Primary syphilis was suspected but darkfield microscopy for Treponema pallidum

Address for reprints: Dr V Ratnam Attili, Dermatovenereology Division, University Teaching Hospital, PO Box 50001, Lusaka, Zambia

Accepted for publication 14 February 1983

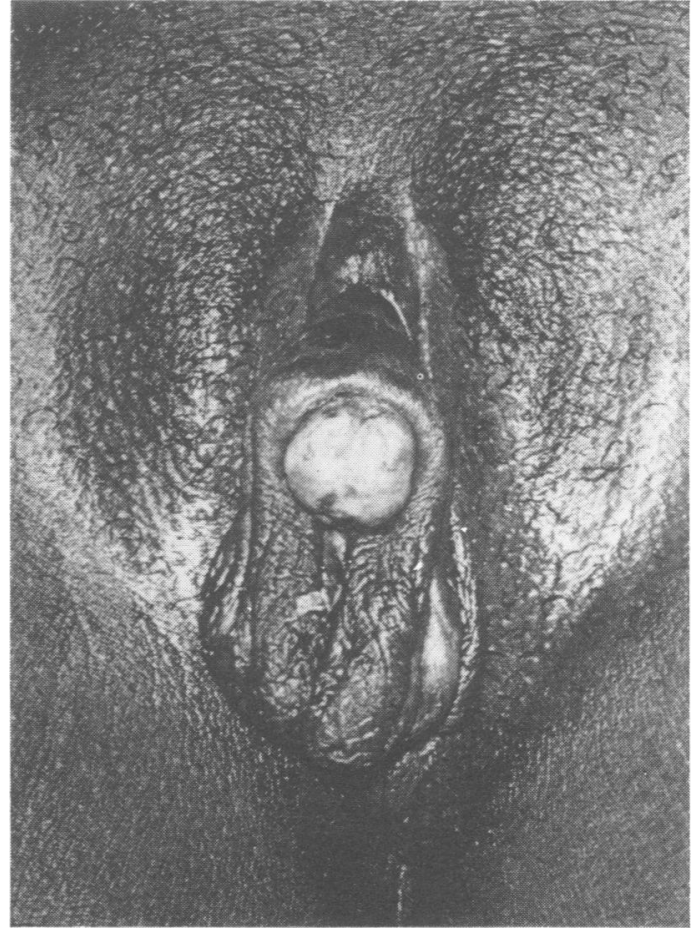

FIG 1 Case 1-Nodulo-ulcerative lesion on the clitoris.

was negative and her serum was non-reactive by the rapid plasma reagin test. Biopsy of the lesion showed numerous $S$ haematobium ova with an intense chronic inflammatory cell reaction in the surrounding skin (fig 2). Although she complained of dysuria, there was no history of haematuria. 


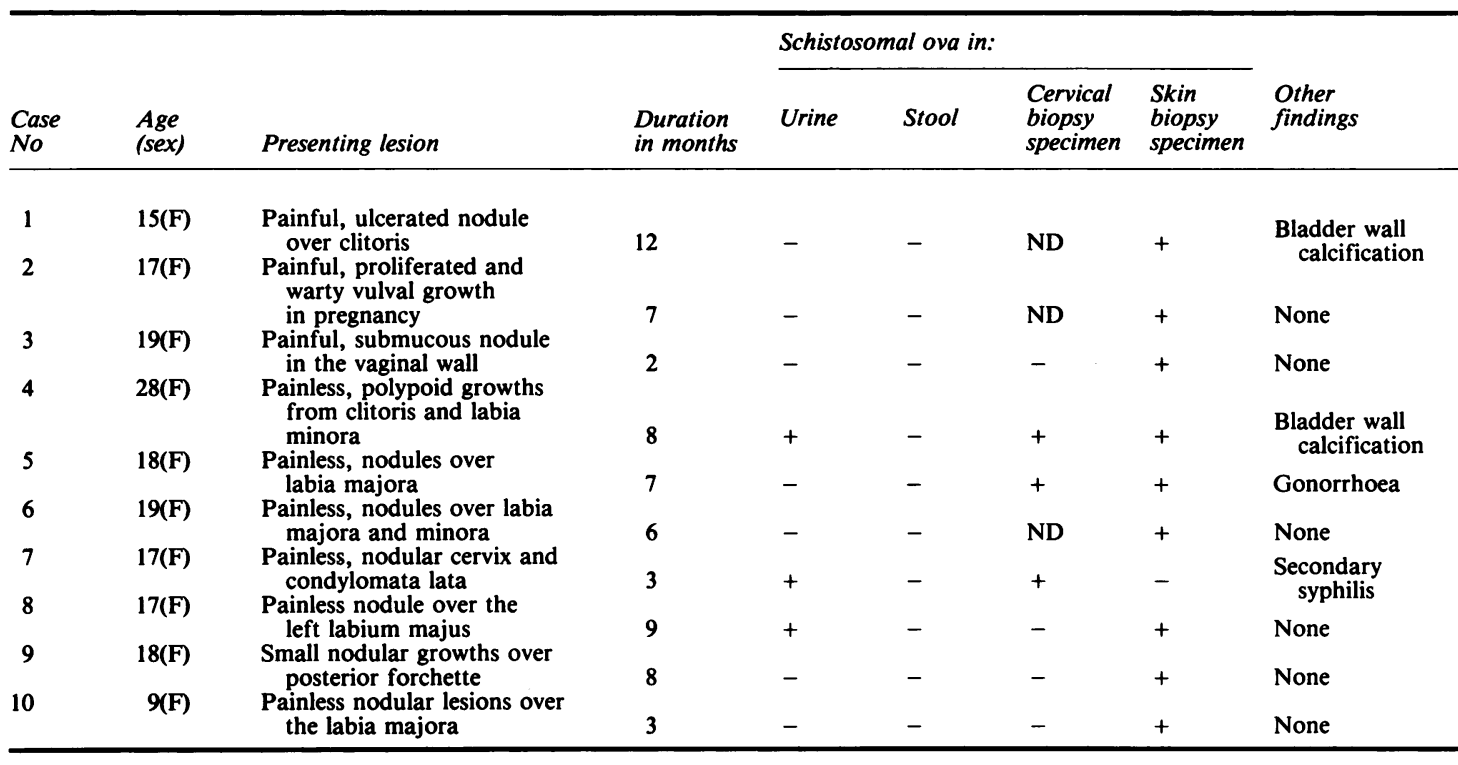

$+=$ positive; - = negative; $\mathrm{ND}=$ not done

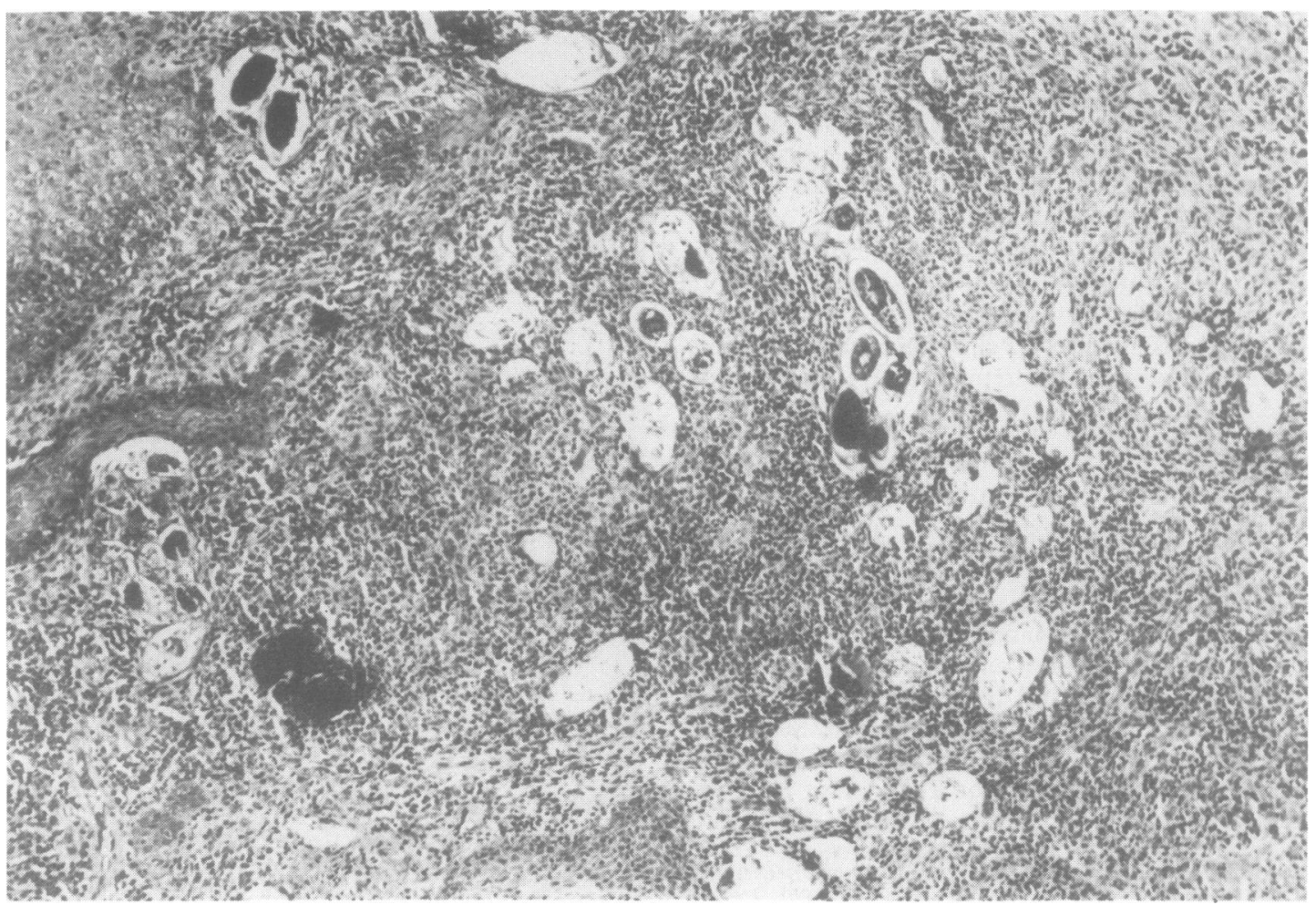

FIG 2 Case 1-Numerous degenerating and calcified S haematobium ova in biopsy tissue. (Haematoxylin and eosin, $\times 40)$. 
Schistosomal ova were not seen in either urine or stool, but there was radiological evidence of extensive calcification in the bladder wall. She was treated with niridazole in a standard regimen, but at review two months later there was no appreciable improvement in the clitoral lesion. She was subsequently lost to follow up.

\section{CASE 2}

A 17 year old married woman, who was seven months pregnant, complained of a rapidly growing painful vulval lesion for seven months. She had a large tender vegetation protruding from the vulval outlet which obstructed speculum examination (fig 3). The surface of the lesion was warty and nodular. She had non-tender bilateral enlargement of the inguinal glands. The clinical diagnosis was of rapidly proliferating genital warts but biopsy of the lesion showed typical schistosomal granulomas with numerous ova. She was lost to follow up before specific treatment could be given.

\section{Discussion}

It is evident from our cases that schistosomal lesions may present a wide variety of appearances (figs 4,5 , 6 , and 7). Oedema and hypertrophy of the affected areas may result from the chronic inflammatory response. Thus the lesions need to be differentiated from genital warts, syphilis, granuloma inguinale, and lymphogranuloma venereum. A definitive diagnosis may be made only if ova are found in the biopsy specimen. The presence of ova in either urine or stool cannot support the diagnosis in endemic

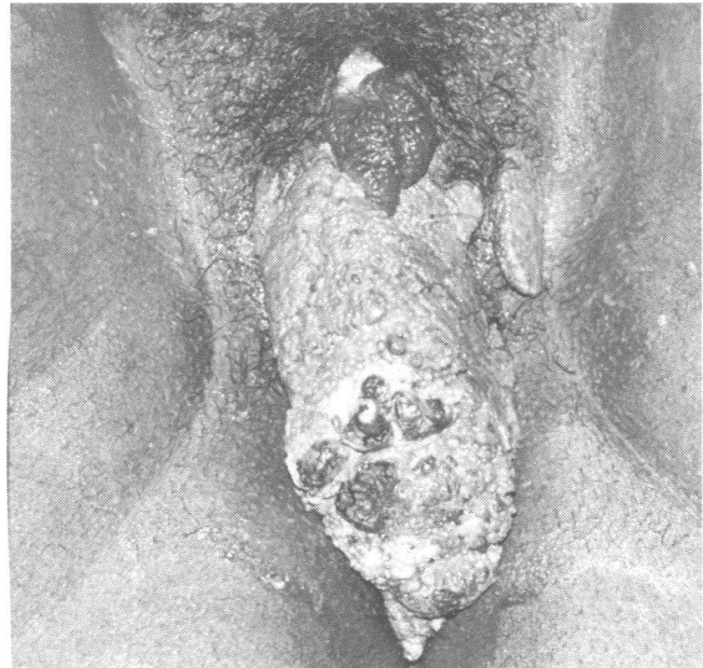

FIG 3 Case 2-Proliferative, warty vulval lesions.

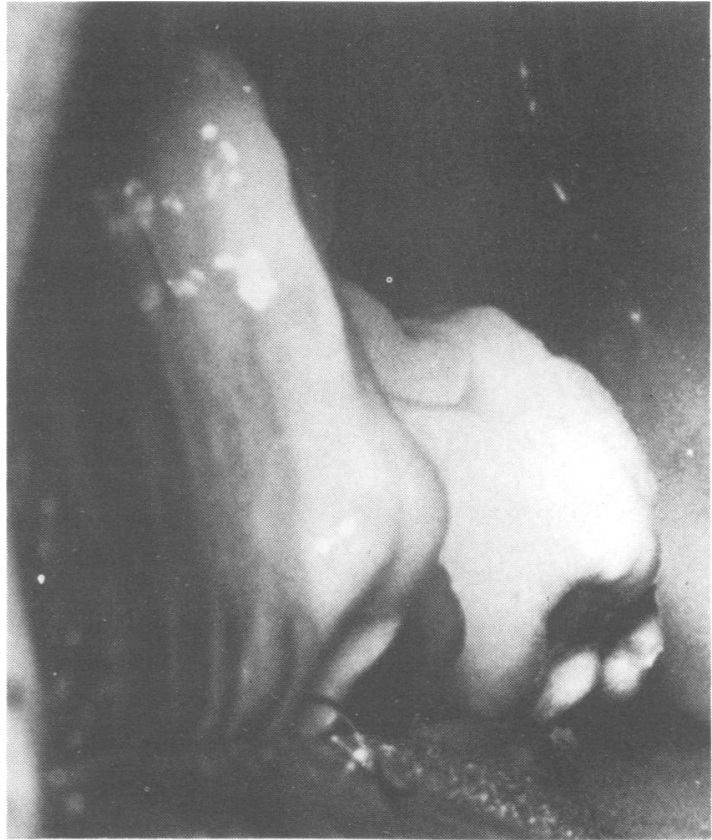

FIG 4 Case 3-Nodular lesion in the vaginal wall.

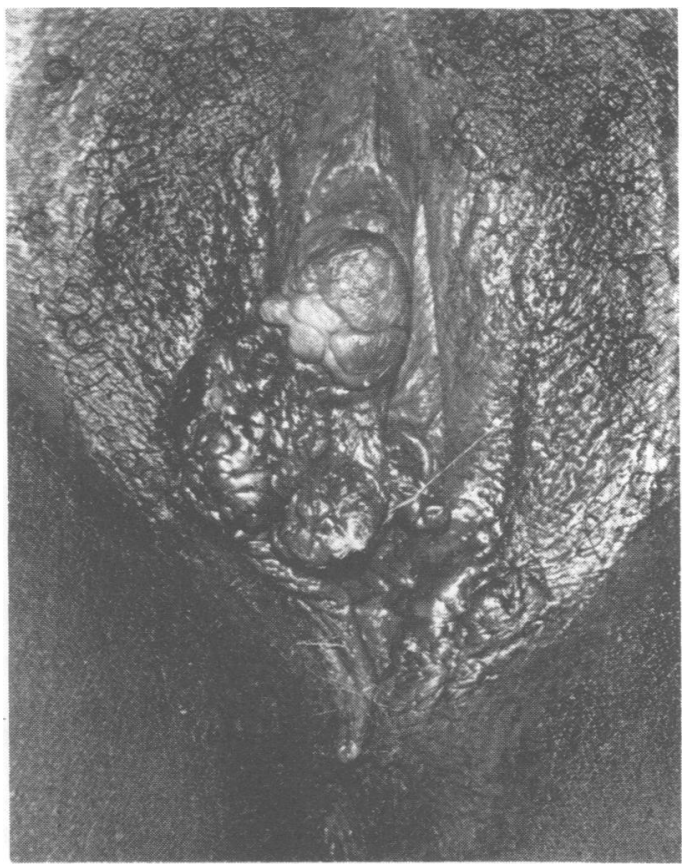

FIG 5 Case 4-Polypoid proliferation of clitoris and labia minora. 


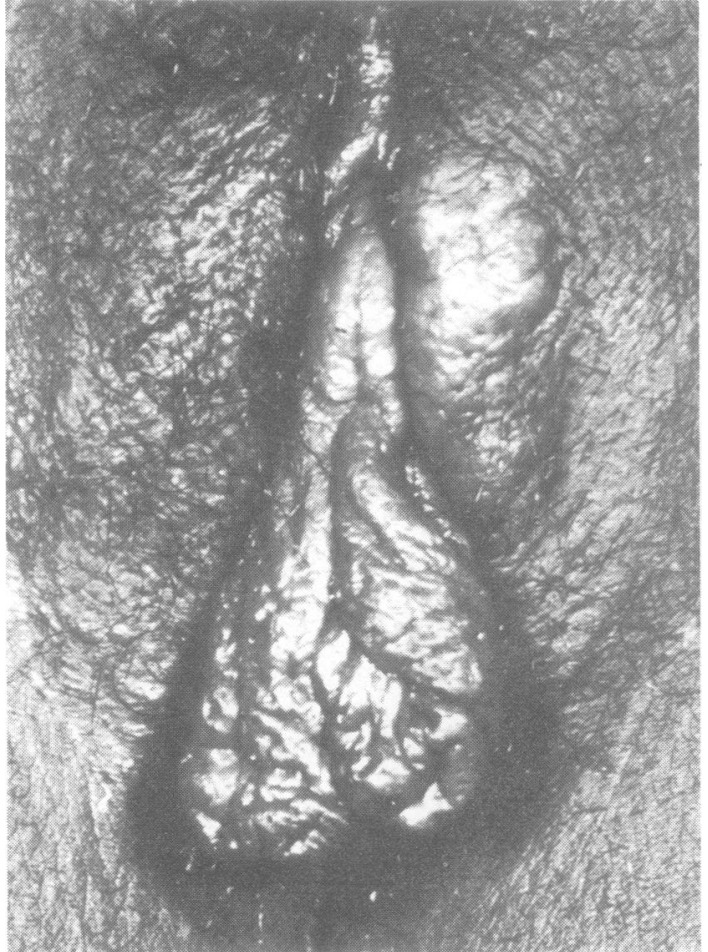

FIG 6 Case 8-Large nodule over left labium majus and hypertrophic labia minora.

areas, since they may often be a coincidental finding. Preponderant association of genital lesions with $S$ haematobium infection and also their more common occurrence in female patients ${ }^{68}$ were confirmed in our study. All our patients were women and ova could be identified in biopsy specimens from nine of them.

Schistosomal genital lesions are not uncommon in endemic areas. Because of the chronicity and painless nature of these lesions patients may not seek early treatment. The diagnosis may easily be missed by an unwary clinician who may not think of performing a biopsy. Many cases may show natural resolution by calcification of ova and fibrosis, but a potential for malignant transformation in chronic cases has been reported. ${ }^{11}$

Earlier reports shed little light on the treatment of genital lesions. El-Zewahry ${ }^{11}$ found improvement after treatment with pentavalent or trivalent antimony preparations and surgery was rarely required. We were less impressed with niridazole treatment in our cases. Chemotherapeutic agents which kill the adult worms undoubtedly stop further flooding of affected tissues with the ova but the skin lesions caused by a granulomatous response to the

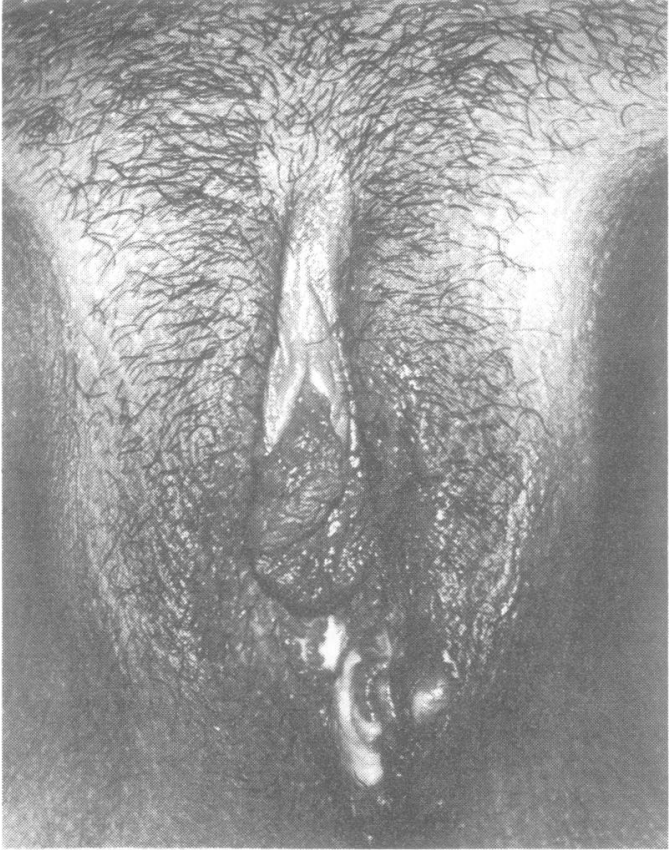

FIG 7 Case 9-Small nodular lesions over the posterior fourchette.

ova that are already present are unlikely to show a rapid response. Natural resolution by calcification and fibrosis may take a long time and esthiomenelike changes in some cases may not be completely reversible.

We thank Mr Ansary of the medical illustration unit for the photographs.

\section{References}

1. Fine J. Studies in bilharzia incidence in Zambia. $E$ Afr Med $J$ 1968; 45:153-5.

2. Hira PR. Snail intermediate hosts and the prevalence of $O$ schistosomiasis in Lusaka, Zambia. Med J Zambia 1975;9;119-24.

3. Handerson AC. Schistosomiasis mansoni. A survey of its incidence at Luampa Hospital. Med J Zambia 1969; 2:165-9.

4. Mampilly J, Sunkwa-Mills HNO. Bilharziasis of the epididymis $\mathrm{N}$ and spermatic cord. Med J Zambia 1976;10:58-9.

5. El-Tayeb AA. Schistosomiasis of the epididymis. Br J Surg N 1969;56:552-3.

6. El-Zeneiny AH, Badawy SZ, Iskandar SG. Bilharzia of the $\mathrm{W}$ female genital tract. J Egypt Med Assoc 1968;5:543-58.

7. Joshi AR. Total granulomatous infarction of testes due to $S$ haematobium. J Clin Pathol 1967; 20:273-5.

8. Berry A. A cytopathological and histopathological study of bilharziasis of the female gential tract. Journal of Pathology and Bacteriology 1966;91:325-38.

9. Adeyemi-Doro FAB, Osoba AO, Junaid TA. Perigenital cutaneous schistosomiasis. Br J Vener Dis 1979;55:446-9.

10. Kingsley HJ. Three cases of cutaneous schistosomiasis of the $\overrightarrow{\mathbb{D}}$ vulva in African women. Cent Afr Med J 1969;12:285-6.

11. El-Zawahry M. Schistosomal granuloma of the skin. $\mathrm{Br} J \mathbb{D}$ Dermatol 1965;11:344-8. 